\title{
Generational cohort effects on trends in the drug overdose mortality epidemic in the United States
}

\author{
Gary G Venter
}

\begin{abstract}
Background: Mortality from drug overdose has increased sharply over recent decades [1]. The purpose of this study is to estimate the variation in overdose mortality across generational cohorts, particularly for white non-hispanic males - a group hit especially hard. Actuaries estimate mortality rates by year-of-birth cohorts for pricing life insurance and annuities. Their Lee-Carter plus cohorts model is applied here to estimate trend by year, pickup rate of trend by age, and cohort impacts on overdose mortality rates in the period 1999-2015 for ages 17-61.
\end{abstract}

Findings: Overdose mortality increased most markedly for the older ages but also for the youngest in this period. Drug mortality for ages 54-61 increased as the pre-boomer generation moved out of this range. The early millennials born 1981-1990 - have sharply higher mortality rates than any other generation after controlling for age and trend. The later millennials are showing much lower overdose mortality. After adjusting for cohort effects, the trend rate was higher in 1999-2007 than in the following years, but 2013-15 gets back towards the higher trend range.

Conclusion: The Lee-Carter plus cohorts model suggests that as they move across the age groups the early millennial cohort will create a bulge in overdose mortality rates that will then subside substantially for the late millennials.

Keywords: Drug overdose; Epidemics; Mortality models; Generations

\section{Background}

Contemporary mortality modeling started with the model of Lee and Carter [2], who model the changes

Correspondence: gv2112@columbia.edu

Full list of author information is available at the end of the article in the $\log$ of the mortality rate by age over time as a beginning mortality curve (mortality rates by age) plus a time trend, which is itself modified by a trend pickup rate varying by age.

In general population mortality, the trend pickup tends to be highest for ages 60 to 80 , as that is where medical improvements have had the greatest impact. For drug overdose mortality in this period, the trend pickup rate was highest for the $55+$ and under 25 ages, with modest trend in the mid-40s group that previously had the highest overdose mortality rates.

In the past dozen or so years actuaries have added year-of-birth cohort effects to the Lee-Carter model. This is a less intuitive component, but appears to have predictive power. Various years of birth show higher or lower mortality at the same age, after adjusting for overall mortality changes over time.

Drivers of cohort effects are still poorly understood, but could relate to: variability in lifetime wealth accumulation across cohorts (highest for the pre-boomers who also show lower mortality); the sheer size of the cohort, especially in relationship to the group slightly older (later boomers got promotions at older ages or never, as they followed a large slightly older group that already had those positions); the state of the economy when entering the workforce; and fighting in wars.

Some of these same drivers - especially economic prospects - could plausibly affect drug overdose mortality as well, which is the motivation for trying the Lee-Carter plus cohorts model here.

\section{Methods}

Data was from the National Center of Health Statistics CDC WONDER age-by-year database for 19992015 [3], for causes of death of unintentional drug poisoning (X40-X44), suicide drug poisoning (X60-X64), homicide drug poisoning (X85), and drug poisoning of undetermined intent (Y10-Y14), as coded in the International Classification of Diseases, 10th Revision.

Overdose mortality rates by age at the beginning, middle and end of the period illustrate the trends. The $\log$ of rates is used and is what is modeled. Because of volatility of individual age mortality rates, the logs of 3 year centered moving averages of rates are used for ages 18-60. 
The Lee-Carter model for mortality rates by age over time sums an initial mortality curve (starting log mortality rates by age) and a log mortality level for each observed time to pick up trend. However the time trend is modified by a pickup rate by age, which provides degrees of participation in the trend across ages. The by-age participation rates do not vary by time in this model, which is often an oversimplification of the process. More general versions of the model allow for multiple trends, each with their own pickup rates [4], but that is a refinement not used at this point.

Actuaries have found that adding year-of-birth cohorts improves the fit and predictive power of the LeeCarter model. To define cohort, year of birth is computed as year of death minus age at death, so is the actual year of birth if death occurs on or after the birthday, and is the following year otherwise. With year of birth $n$ and age $u$, the Lee-Carter plus cohorts model for $y[n, u]$, the log of the mortality rate, is:

$$
y[n, u]=c+p[n]+q[u]+r[n+u] s[u]+\epsilon[n, u](1)
$$

Here $\mathrm{c}$ is the constant term, $q[u]$ is the initial mortality curve at age $u, r[n+u]$ is the trended overall mortality level at year of death $n+u, s[u]$ is the trend pickup rate at age $u, p[n]$ is the year of birth adjustment, which is constant over time for any given year of birth $n$, and $\epsilon[n, u]$ is the stochastic component. In this application, there is a $p$ parameter for each year of birth 1938-98, a $q$ and an $s$ parameter for each age 17-61, and a levelafter-trend parameter $r$ for each year 1999-2015.

For identifiability $r$ and $q$ are set to zero at their first observations, and to keep trends and cohort effects separate $p$ is forced to have no trend by subtracting a fitted trend line. The pickup rate $s$ is forced to be positive with a maximum at 1 . Thus the trend is expressed as the maximum trend for any age, with pickup rates $\leq 1$ at other ages.

Lasso-like parameter shrinkage [5] is used to reduce the effective number of parameters by a Bayesian MCMC estimation procedure. Each parameter type $p, q, r, s$ is fit on a curve expressed as a series of linear steps by year, with the changes in slope at each point shrunk towards zero by giving each change a meanzero double exponential (Laplace) prior with a small standard deviation. This was implemented in the Stan package developed by the Columbia University Statistics Department [6].

\section{Findings}

Figure 1 is a graph of log overdose mortality by age for 1999, 2007, and 2015. To smooth fluctuations, each point is the log of the centered three-year moving average overdose mortality rate at the age shown. The greatest increases were at the oldest and youngest ages, with more moderate increases for ages in the mid 40s.

The estimated values for the four types of parameters $p, q, r$, and $s$ are in Table 1 . The trend-pickup-by-age parameters $s$ are forced to have a maximum of 1.0 in every sample, but that maximum can occur at different ages in different samples, so the posterior mean is not 1.0 at any age. The oldest and youngest ages are getting the highest trend pickup, with mid-40s ages getting about a quarter of the overall trend.

The modeled annual change in trend $\Delta r$ is shown instead of $r$. The trend moderated after 2007, but increased in the most recent years.

Figure 2 graphs the cohort parameters $p$ for years of birth 1938-1995. The parameters for 1996-8 continue the downward curve on the right, but the data for them makes their estimates uncertain. This is the case for 1938-40 as well. The pre-boomer generation shows relatively low mortality here, as does the late millennial group born 1991+. There is a bit of a dip from 1963-73, corresponding largely to Gen X. The early millennials have the highest mortality parameters.

For perspective, the actual log mortality at age 45 increased from 1.11 in 1999 to 1.33 in 2015. All the other ages had greater increases. The cohort $p$ was 0.08 for 1954-57 years of birth, compared to 0.04 for 196870. This 0.04 difference would be a noticeable increase to the age 45 cumulative change of 0.22 . For $1980-81$ years of birth, $p$ was 0.14 , so 0.1 higher than the 1968 70 level. This is greater than the 2015 excess of the early 30s ages log mortality rates over those for the mid 40s. The $1943 p$ was about 0.25 less than that for the mid-1950s group, and that is about a third of the increase in log mortality at age 57 from 1999 to 2015.

\section{Discussion}

Figure 1 shows that the change in overdose mortality rates was highest at the oldest ages, and was also high at the youngest ages. In response, the estimates of the modeled pickup rates for trend are highest at these ages. In 1999, ages 54-61 were from the more traditional pre-boomer generation, and some of the trend in Figure 1 is likely to be due to them being replaced by the boomers during the period. The cohort parameters reflect this difference, accounting for some of the trend change.

The relatively modest trend for ages in the mid $40 \mathrm{~s}$ shows up in the model as lower trend pickup rates for those ages. By 2015 Gen X had replaced the boomers in this age range, and the lower estimated mortality for the Gen X cohorts provides an explanation for the small increase for these ages in the last 8 years, as shown in Figure 1. 


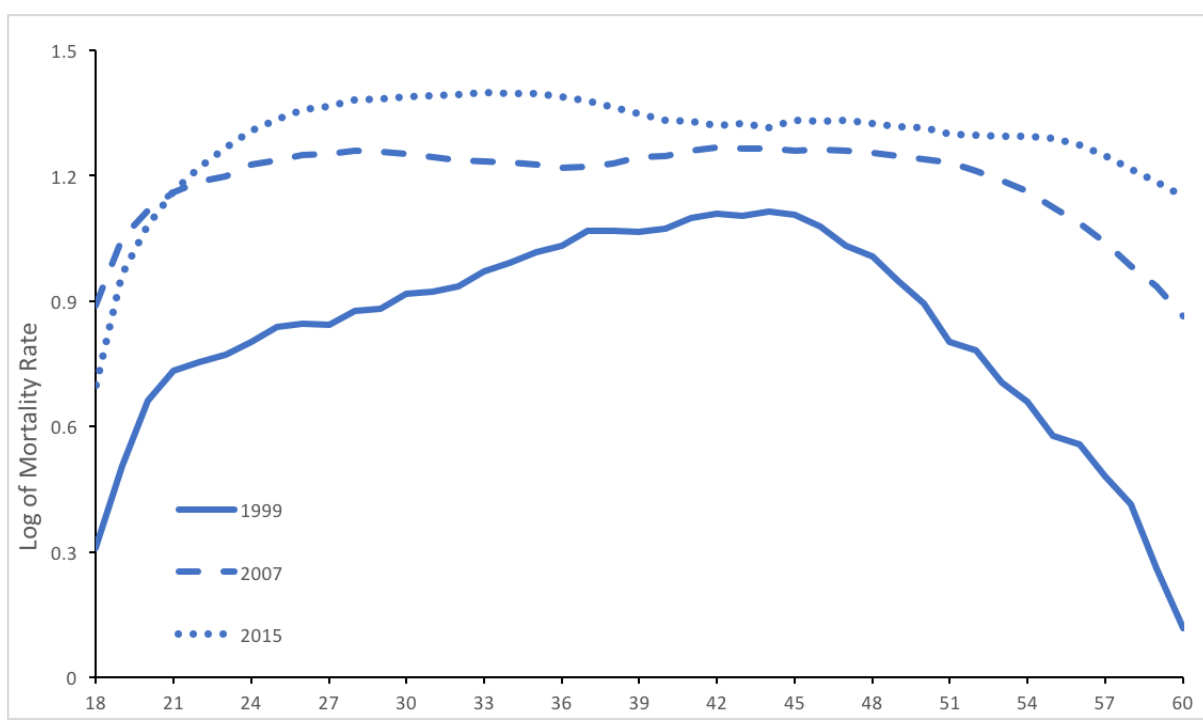

Figure 1 Log of overdose mortality by age for 1999, 2007, and 2015, three-year moving average

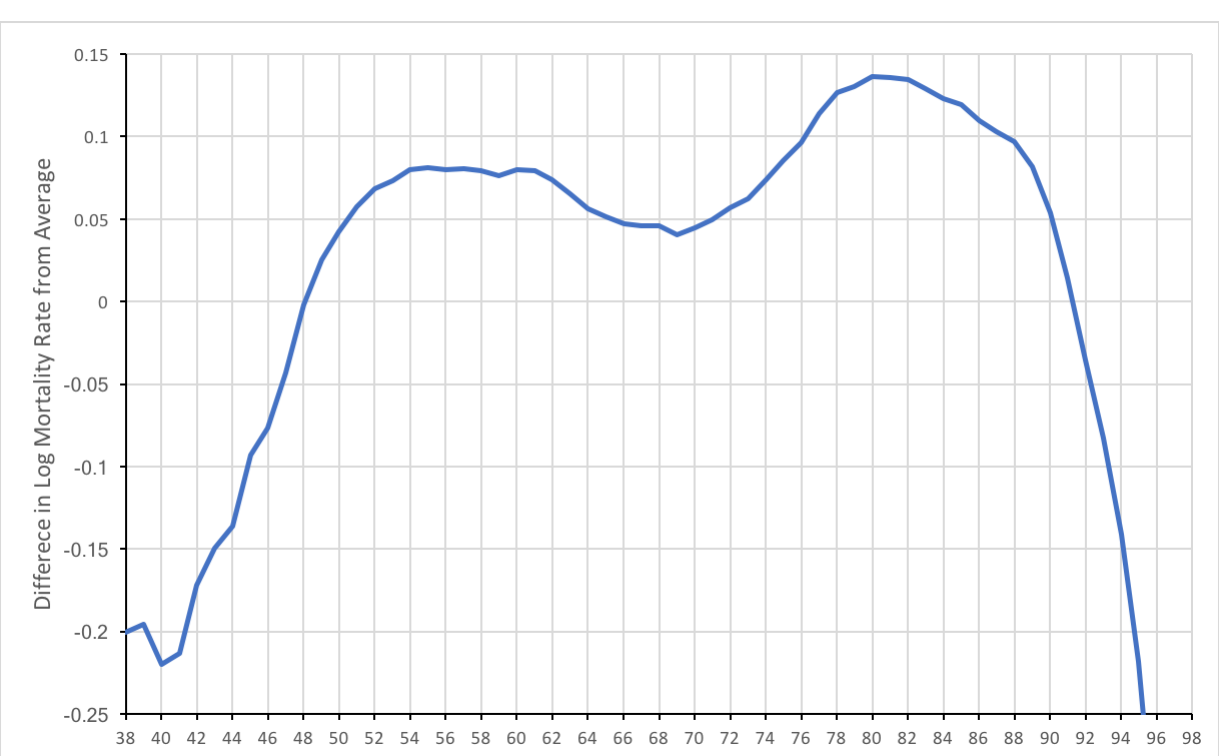

Figure 2 Cohort parameters $p$ for years of birth 1938-95 
In 1999, age 33 was from the boomer generation, in 2007 from Gen X, and in 2015 from the early millennials. By 2015, ages in the 30s had replaced those in the 40 s as the group with the highest overdose mortality. This is accounted for in the model by the cohort parameter differences for Gen X and the early millennials. The bulge in mortality rates seen in Figure 1 around age 27 for 2007 and age 35 for 2015 both come from year of birth 1980, which has the highest fitted cohort parameter. Figure 1 also shows that overdose mortality for ages in the late teens dropped from 2007 to 2015 . The late millennials replaced the early millennials here and have lower cohort parameters.

People born in different time periods face different demographic, economic, and military conditions. Anything that affects the economic well being of these groups plausibly affects mortality, including overdose mortality. E.g., see [7]. The pre-boomer group, for instance, had the highest lifetime wealth, adjusted for inflation, in history [4]. They benefited from being a relatively small group followed by a larger group, which facilitated opportunities for managerial appointments. They entered the workforce during a good economy, and were too old for the Vietnam draft. The boomers had the opposite experience in each category, and Gen $\mathrm{X}$ was in-between. The early millennials are a larger generation, they fought the Mideast wars, and faced the great recession early in their careers. The laterborn millennials entered the workforce in a more favorable environment and in a time of less military engagement. All these influences plausibly affect overdose mortality. More research is needed to fully comprehend such trends.

\section{Conclusions}

This model is silent on future trends, and trend pickup by age might flatten out now that the overdose mortality rates have. In fact now that overdose mortality rates are fairly flat by age, in the next few years the overall trend might come down to more resemble the age 43 trend, which was about a quarter of the overall level in this period. Boomers aging out of the 17-61 age range and being replaced by the recent generation would put downward pressure on the overall rates, according to this model. However the early millennials could have even higher overdose mortality rates as they age into their 40 s, which would be an opposing effect.

Numerous intervention programs for preventing and treating drug overdose have been developed or are under development, including prescription monitoring, training on rescue treatments, and community-based interventions. It would appear that focusing intervention on the population born in the 1980s would be fruitful.
References

1. Rudd, R.A., Seth, P., David, F., Scholl, L.: Increases in drug and opioid-involved overdose deaths - United States, 2000-2015. Morbidity and Mortality Weekly Report 65(5051), 1445-1452 (2016)

2. Lee, R.D., Carter, L.R.: Modeling and forecasting U.S. mortality. Journal of the American Statistical Association 87, 659-675 (1992)

3. CDC: Wide-ranging online data for epidemiologic research (WONDER). CDC, National Center for Health Statistics Atlanta GA (https://wonder.cdc.gov)

4. Howe, N.: Generations in pursuit of the American Dream. http://www.lifecourse.com/assets/files/reports/Generations\%20in $\% 20$ Pursuit $\% 20$ of \%20the\%20American\%20Dream.pdf (2014)

5. Li, H., Pati, D.: Variable selection using shrinkage priors. arXiv:1503.04303v2 [stat.ME] 22 Mar2015 (https://arxiv.org/pdf/1503.04303.pdf)

6. Carpenter, B., Gelman, A., Hoffman, M.D., Lee, D., Goodrich, B., Betancourt, M., Brubaker, M.A., Guo, J., Li, P., Riddell, A.: Stan: A probabilistic programming language. Journal of Statistical Software 76:1 (January 2017)

7. Case, A., Deaton, S.A.: Mortality and morbidity in the 21st century. Brookings Papers on Economic Activity Spring 2017 (2017)

Tables

Table 1 Parameters by Age, Year-of-Birth Cohort, and Year

$\begin{array}{ccccccc}\text { Age } & \mathrm{q} & \mathrm{s} & \text { Cohort } & \mathrm{p} & \text { Cohort } & \mathrm{p} \\ 17 & 0 & 0.681 & 1938 & -0.201 & 1983 & 0.129 \\ 18 & 0.224 & 0.905 & 1939 & -0.196 & 1984 & 0.123 \\ 19 & 0.438 & 0.753 & 1940 & -0.220 & 1985 & 0.119 \\ 20 & 0.543 & 0.678 & 1941 & -0.213 & 1986 & 0.109 \\ 21 & 0.617 & 0.625 & 1942 & -0.172 & 1987 & 0.103 \\ 22 & 0.654 & 0.594 & 1943 & -0.149 & 1988 & 0.097 \\ 23 & 0.688 & 0.568 & 1944 & -0.136 & 1989 & 0.082 \\ 24 & 0.719 & 0.542 & 1945 & -0.093 & 1990 & 0.054 \\ 25 & 0.740 & 0.520 & 1946 & -0.077 & 1991 & 0.014 \\ 26 & 0.759 & 0.508 & 1947 & -0.043 & 1992 & -0.035 \\ 27 & 0.775 & 0.492 & 1948 & -0.002 & 1993 & -0.083 \\ 28 & 0.787 & 0.477 & 1949 & 0.025 & 1994 & -0.141 \\ 29 & 0.807 & 0.462 & 1950 & 0.042 & 1995 & -0.218 \\ 30 & 0.821 & 0.438 & 1951 & 0.057 & 1996 & -0.338 \\ 31 & 0.841 & 0.422 & 1952 & 0.068 & 1997 & -0.475 \\ 32 & 0.861 & 0.397 & 1953 & 0.073 & 1998 & -0.614 \\ 33 & 0.882 & 0.371 & 1954 & 0.080 & & \\ 34 & 0.902 & 0.349 & 1955 & 0.081 & \text { Year } & \Delta \mathrm{r} \\ 35 & 0.922 & 0.331 & 1956 & 0.080 & 2000 & 7.3 \% \\ 36 & 0.938 & 0.309 & 1957 & 0.080 & 2001 & 8.5 \% \\ 37 & 0.959 & 0.295 & 1958 & 0.080 & 2002 & 17.9 \% \\ 38 & 0.973 & 0.284 & 1959 & 0.076 & 2003 & 9.1 \% \\ 39 & 0.974 & 0.280 & 1960 & 0.080 & 2004 & 5.7 \% \\ 40 & 0.984 & 0.274 & 1961 & 0.079 & 2005 & 6.2 \% \\ 41 & 0.993 & 0.270 & 1962 & 0.074 & 2006 & 7.1 \% \\ 42 & 0.993 & 0.264 & 1963 & 0.065 & 2007 & 5.0 \% \\ 43 & 0.995 & 0.255 & 1964 & 0.056 & 2008 & 1.3 \% \\ 44 & 0.996 & 0.264 & 1965 & 0.051 & 2009 & 0.7 \% \\ 45 & 0.988 & 0.280 & 1966 & 0.047 & 2010 & 2.2 \% \\ 46 & 0.971 & 0.308 & 1967 & 0.046 & 2011 & 2.8 \% \\ 47 & 0.948 & 0.335 & 1968 & 0.046 & 2012 & 1.7 \% \\ 48 & 0.920 & 0.358 & 1969 & 0.040 & 2013 & 3.5 \% \\ 49 & 0.889 & 0.385 & 1970 & 0.045 & 2014 & 5.2 \% \\ 50 & 0.856 & 0.414 & 1971 & 0.049 & 2015 & 7.3 \% \\ 51 & 0.830 & 0.443 & 1972 & 0.057 & & \\ 52 & 0.805 & 0.448 & 1973 & 0.063 & \text { Constant } & 0.0298 \\ 53 & 0.777 & 0.468 & 1974 & 0.074 & & \end{array}$

0.119

0.097

0.082

0.035

083

0.218

0.338

0.475

$\Delta r$

$7.3 \%$

$17.9 \%$

$9.1 \%$

$5.7 \%$

$.2 \%$

$.0 \%$

$1.3 \%$

$0.7 \%$

$1.7 \%$

$.5 \%$

$.2 \%$

0.0298 